\title{
End-Systolic Pressure-Diameter Relation of the Left Ventricle during Transient and Sustained Elevations of Blood Pressure
}

\author{
Edson Antonio Bregagnollo, Katashi O koshi, Beatriz Bojikian Matsubara, Paulo José Ferreira Tucci
}

Botucatu, SP - Brazil

\begin{abstract}
Objective - To assess the effect of transient and sustained variations in cardiac load on the values of the end-systolic pressure-diameter relation (ESPDR) of the left ventricle.

Methods - We studied 13 dogs under general anesthesia and autonomic blockade. Variations of cardiac loads were done by elevation of blood pressure by mechanical constriction of the aorta. Two protocols were used in each animal: gradual peaking and decreasing pressure variation, the "transient arterial hypertension protocol" (TAH), and a quick and 10 min sustained elevation, the "sustained arterial hypertension protocol"(SAH). Then, we compared the ESDR in these two situations.
\end{abstract}

Results - Acute elevation of arterial pressure, being it "transitory" or "sustained", did not alter the heart frequency and increased similarly the preload and after load. However, they acted differently in end systolic pressurediameter relation. It was greater in the SAH than TAH protocol, $21.0 \pm 7.3 \mathrm{mmHg} / \mathrm{mm} v s .9 .2 \pm 1.2 \mathrm{mmHg} / \mathrm{mm}(p<0.05)$.

Conclusion - The left ventricular ESPDR values determined during sustained pressure elevations were higher than those found during transient pressure elevations. The time-dependent activation of myocardial contractility associated with the Frank-Starling mechanism is the major factor in inotropic stimulation during sustained elevations of blood pressure, determining an increase in the ESPDR values.

Key words: end-systolic pressure-diameter relations, cardiac inotropism, arterial hypertension

Faculdade de Medicina de Botucatu - UNESP

Mailing address: Edson Antonio Bregagnollo - Depto. de Clínica Médica -

Faculdade de Medicina de Botucatu - UNESP - Rua Rubião Jr, S/N - 18618-000

- Botucatu, SP, Brazil

English version by Stela Maris C. Gandour
Studies by Suga et al in the " 70 s have shown that in preparations of isolated ejecting hearts the values of left ventricular pressure-volume relations $(\mathrm{P} / \mathrm{V})$ taken in isochronic points of heartbeats occurring in different load conditions have a linear relation. As time passes, from the start of the ventricular contraction, these linear relations constitute a set of straight lines with progressively increasing inclinations, reaching a maximum value at the end of systole. Considering that this approach showed alterations in the elastic characteristics of the ventricular chamber with respect to time, the investigators applied the concept of the pressure-diameter relation varying in time to the ventricle ${ }^{1-5}$. According to Suga et $\mathrm{al}^{1-4}$, the inclination of the straight line determined by the $\mathrm{P} / \mathrm{V}$ relations at the end of systole did not change with wide variations in pre- and afterload, but was, however, extremely sensitive to alterations in the contractile state. Based on these observations, these authors proposed the use of the inclination of this straight line as an index of myocardial contractility called the end-systolic pressure-diameter relation. Later, other authors ${ }^{6-8}$ also validated the end-systolic pressure-diameter relation as a safe method for evaluating the inotropic state of the cardiac muscle. On the other hand, several studies on intact hearts ${ }^{9-15}$ have shown that the $\mathrm{P} / \mathrm{V}$ relations at the end of systole may depend on the conditions of the cardiac load. Therefore, the end-systolic P/V relations of ejecting heartbeats do not coincide with those observed in isovolumetric beats.

Another point to be considered is that studies about myocardial contractility performed on isolated cardiac muscles ${ }^{16-20}$, isolated hearts ${ }^{21,22}$, and in situ hearts ${ }^{23}$ have shown a constant interaction between the length of the cardiac fiber at rest and the contractile state of the myocardium.

Due to these conflicts found in the literature, this study aimed to verify if in the in situ heart the cardiac load conditions modify the values of end-systolic pressure-diameter relation. We assessed whether the end-systolic pressure-diameter relation values determined during transient elevations of blood pressure were equivalent to those determined during sustained elevations of blood pressure when time-dependent cardiac adjustments had already be- 
en completed. These cardiac adjustments are triggered by arterial hypertension and result from the constant interaction between the preload and afterload present in the in situ heart $^{24}$.

\section{Methods}

Thirteen male dogs with body weights ranging from 20 to $35 \mathrm{~kg}$ underwent anesthesia with intravenous chloralose $(60 \mathrm{mg} / \mathrm{kg})$ and urethane $(600 \mathrm{mg} / \mathrm{kg})$. After anesthesia, the animals were put in the dorsal decubitus position, intubated, and mechanically ventilated with the Takaoka ${ }^{\mathrm{TM}}$ ventilator, at 16 cycles per minute. The femoral vein was dissected and cannulated for drug administration and volume repositioning.

After median sternotomy, the aorta was dissected and repaired with a cardiac band immediately after the emergence of the left subclavian artery. The pericardium was opened, and the margins of the incision were fixed in the anterior chest wall to maintain the heart in a stable position. To obtain left ventricular pressures, a catheter of the OdmanLedin type (length: $4 \mathrm{~cm}$; internal diameter: $1.4 \mathrm{~mm}$ ) was inserted into the left ventricular cavity through a puncture in the apical region. After the surgical procedures, the dogs underwent heparinization (5000 IU/kg, intravenously). A manometric system comprising a P23ID Stathan transducer coupled with a 4 RFO Funbec amplifier was used for recording the left ventricular pressures. The dynamic characteristics of this system had already been assessed in our laboratory and were considered adequate for the assessments performed ${ }^{25}$. The horizontal plane passing through the middle of the left ventricular cavity was used as reference zero. The M-mode echocardiogram of the left ventricular cavity was performed with a 5-MHz transducer located on the free wall of the right ventricle, and the ultrasound beam was directed perpendicularly to the interventricular septum and immediately below the mitral valve plane. This arrangement was used for determining the variations in the greatest left ventricular cross-sectional diameter, according to the recommendations of the ISFC/WHO ${ }^{26}$.

To avoid the influence of the fluctuation of the autonomic activity upon the cardiac inotropism, the dogs underwent a parasympathetic (atropine: $0.5 \mathrm{mg} / \mathrm{kg}$, intravenously) and beta-adrenergic (oxprenolol: $3 \mathrm{mg} / \mathrm{kg}$, intravenously) blockade. The efficacy of this blockade had previously been tested in our laboratory ${ }^{27}$. Before applying the experimental protocols, a 10-minute period was awaited for evaluating the stability of the preparation.

In all animals studied, two experimental protocols were applied. The transient and sustained arterial hypertension protocols are schematically represented in figure 1 . In both protocols, blood pressure was elevated pulling the cardiac band repairing the aorta. In the transient arterial hypertension protocol, tension was gradually exerted to create an aortic constriction capable of progressively increasing blood pressure to approximately $50 \mathrm{mmHg}$ above the basal levels (To condition) during 4 to 6 heartbeats. At the time

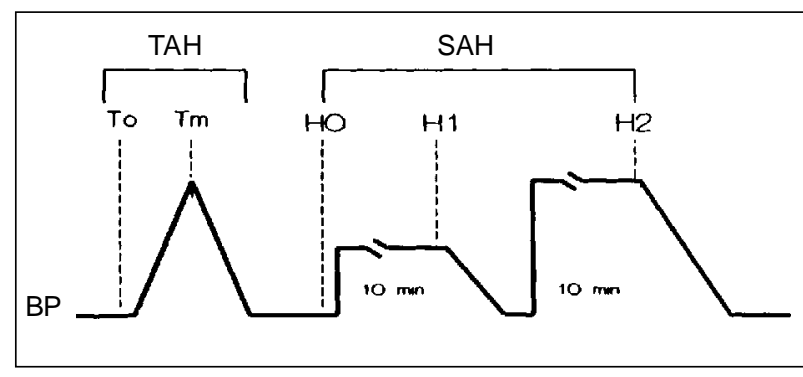

Fig. 1-Sketch of the experimental protocols: BP- blood pressure; TAH- transient arterial hypertension; $\mathrm{SAH}-$ sustained arterial hypertension; To and Tm: basal and maximum BP condition in the TAH protocol; $\mathrm{H}_{0} \mathrm{H}_{1}$ e $\mathrm{H}_{2}$ - basal, hypertensive 1 and hypertensive 2 conditions of the SAH protocol, respectively.

blood pressure reached the desired maximum value (Tm condition), the aortic constriction was gradually released to bring blood pressure to basal levels. During these procedures, simultaneous recordings of the electrocardiographic II lead of the left ventricle were taken, as were the recordings of the left ventricular pressure curve and echocardiogram for further determination of the values of the end-systolic pressure-diameter relation (fig. 2). In the sustained arterial hypertension protocol after recording the variables at the basal level (Ho), the blood pressure was elevated by the same maneuver in two steps. In the first step, an aortic constriction was established to increase blood pressure by approximately $25 \mathrm{mmHg}$ and to sustain it elevated for 10 minutes (hypertensive condition $1-\mathrm{H} 1$ ). The aortic constriction was then released and the cardiac band again tensioned to establish an aortic constriction to increase blood pressure to approximately $50 \mathrm{mmHg}$ above the Ho levels for 10 minutes (hypertensive condition 2: H2). In the experimental conditions $\mathrm{Ho}, \mathrm{H} 1$, and $\mathrm{H} 2$, simultaneous recordings of the left ventricular electrocardiographic II lead, pressure curve and echocardiogram were also performed for further determination of the values of the end-systolic pressurediameter relation (fig. 3). All recordings were obtained after 10 minutes of expiratory apnea to eliminate interferences of respiration upon pressure and cardiac dimensions.

The recordings of the left ventricular echocardiogram, pressure curve, and electrocardiogram were calibrated with the aid of a computed system comprising a version 3 Digi-

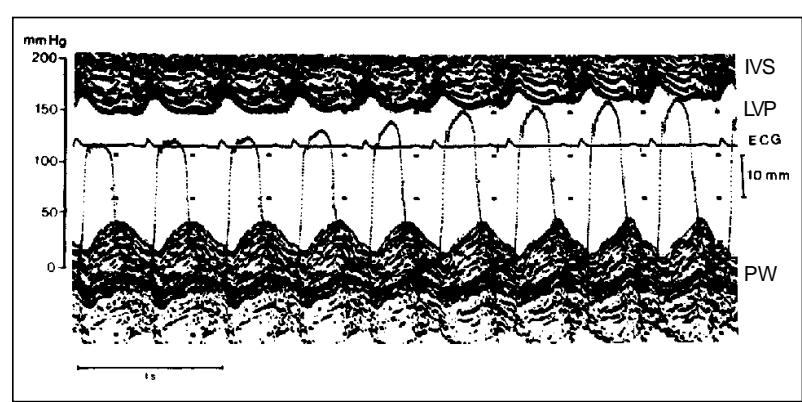

Fig. 2-Tracing of the simultaneous electrocardiographic recording (ECG); left ventricular pressure (LVP) and left ventricular echocardiogram (IVS- interventricular septum; PW-posterior wall) obtained during transient elevations of blood pressure. 


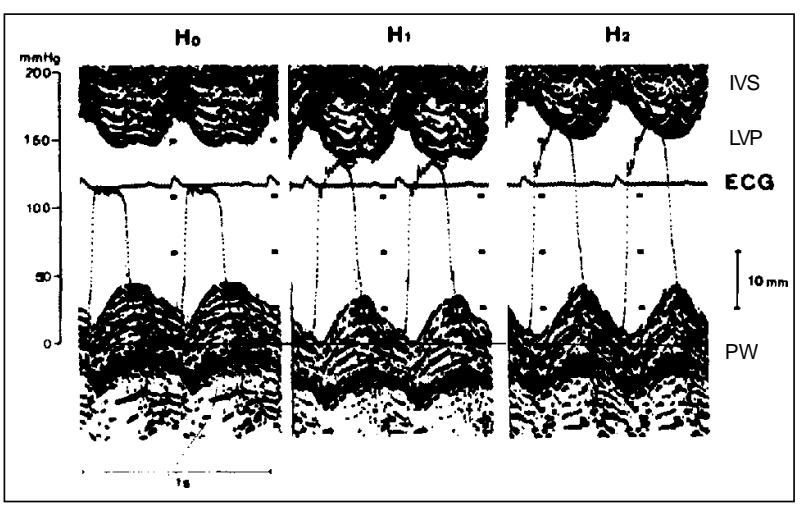

Fig. 3 - Tracing of the simultaneous electrocardiographic recording (ECG); left ventricular pressure (LVP) and left ventricular echocardiogram (IVS- interventricular septum; PW- posterior wall) obtained during sustained elevations of blood pressure.

graph Renoir digitizing table, a PC-286 Itautec computer, and software designed by the Fundação para o Desenvolvimento Tecnológico e Engenharia (FDTE) of the Escola Politécnica of the Universidade de São Paulo. In this program, during the entire cardiac cycle, the following parameters were calculated every five miliseconds: left ventricular pressure (P); left ventricular cross-sectional diameter (D); thickness of the left ventricular posterior wall (h); and also the instantaneous mathematical relation between the values of $P$ and $D$. The $P$ values were projected in the axis of the ordinates and the D values in the axis of the abscissas of the Cartesian system of axes. This allowed the graphic representation of the cardiac cycles by P/D loops. The time that the P/D relation reached the maximum value was considered the end of systole ${ }^{28}$ and was marked with an $\mathrm{X}$ in the P/D loop, and its values were taken as end-systolic pressure (ESP) and diameter (ESD). In each experimental situation, the ESP and ESD values of heartbeats occurring in different load conditions were taken as coordinates for obtaining the straight lines of simple linear regression ${ }^{29}$. The angular coefficient of the straight lines obtained defined the values of the endsystolic pressure-diameter relation in each experimental condition. In the transient arterial hypertension protocol, the end-systolic pressure-diameter relation values (ESPDRTE) were obtained from 4 or 5 cardiac cycles occurring in different load conditions during a transient elevation in blood pressure. In the sustained arterial hypertension protocol, the end-systolic pressure-diameter relation values (ESPDR$\mathrm{SE}$ ) were obtained from the linear relations between the endsystolic points of heartbeats occurring during blood pressure stability at the end of the $\mathrm{Ho}, \mathrm{H} 1$, and $\mathrm{H} 2$ conditions. In each experimental condition, the mean values of ESP and ESD considering 5 consecutive cardiac cycles were used as coordinates for determining the straight lines of simple linear regression whose angular coefficient defined the ESPDR-SE value for each dog studied. For each simple linear regression, the Pearson correlation coefficient (r) was calculated between the points used for determining the respective straight lines. In the transient and sustained arterial hypertension protocols, in addition to the end-systolic pressure-diameter relation, the behavior of the following variables was also assessed: left ventricular systolic (LVSP) and end-diastolic pressures (LVEDP), left ventricular systolic (SD) and diastolic (DD) diameters, systolic (Sh) and diastolic (Dh) thickness of the left ventricular posterior wall, heart rate (HR). In the transient arterial hypertension protocol, the values of these variables at the basal level (To) were compared with those verified when the blood pressure reached the maximum value (Tm). In the sustained arterial hypertension protocol, the values of these variables were compared in the $\mathrm{Ho}, \mathrm{H} 1$ and $\mathrm{H} 2$ conditions. For all variables studied, the values found in the To and Tm conditions of the transient arterial hypertension protocol were compared with those found in the Ho and $\mathrm{H} 2$ conditions, respectively, of the sustained arterial hypertension protocol.

\section{Results}

Preliminary analyses of descriptive statistics showed that the ESPDR did not have a gaussian distribution. For this reason, the comparisons of the ESPDR-SE and ESPDRTE values were performed using the Friedman test ${ }^{30}$. For the other variables (LVSP, LVEDP, SD, DD, Sh, Dh, HR), we used the analysis of repetitive measures (Morrison, 1976) complemented by the analysis of confidence intervals for interest contrasts $(\mathrm{CI}=95 \%)$. The differences between the compared values were considered significant when the statistical probability by chance was lower than $5 \%$ $(\mathrm{p}<0.05)$.

Table I shows the values $\left({ }^{-} \mathrm{x} \pm \mathrm{sd}\right)$ of the variables analyzed with the use of the transient and sustained arterial hypertension protocols. The statistical analyses used to compare these results allowed us to make the following observations: 1) the heart rate did not significantly vary; 2) the maximum increases in LVSP and LVEDP were similar; 3) the variations in left ventricular SD and DD were statistically different; 4) the alterations in Sh and Dh of the left ventricular posterior wall were similar. These results show that the alterations in cardiac load triggered by elevation in pressure were similar in both protocols, being, however, transient or sustained. Comparison of load conditions (LVSP, LVEDP, $\mathrm{SD}, \mathrm{DD}, \mathrm{Sh}, \mathrm{Dh}$ ) in basal (To and Ho) and maximum (Tm and $\mathrm{H} 2$ ) conditions in both protocols did not also identify significant differences between them. These observations lead to the conclusion that alterations in cardiac loads triggered by hypertension were similar in the transient and sustained arterial hypertension protocols.

The graphic representation of the cardiac cycles by the P/D loops using the software developed by the FDTE reproduced those reported in the literature ${ }^{31-33}$. Figure 4 illustrates $3 \mathrm{P} / \mathrm{D}$ loops obtained in one experiment. In each loop, the time of the cardiac cycle when the $\mathrm{P} / \mathrm{D}$ relation reaches the maximum value is marked with an $\mathrm{X}$ and identifies the end of systole in each cardiac cycle. The linear relation between the end-systolic points is the coordinate that de- 


\begin{tabular}{|c|c|c|c|c|c|}
\hline & \multicolumn{2}{|c|}{$\mathrm{TAH}$} & \multicolumn{3}{|c|}{ SAH } \\
\hline & To & $\mathrm{Tm}$ & Но & H1 & $\mathrm{H} 2$ \\
\hline HR (bpm) & $136 \pm 22$ & $137 \pm 23^{\text {(a) }}$ & $136 \pm 25^{\text {(a) }}$ & $136 \pm 23^{(a)}$ & $136 \pm 24^{(a)}$ \\
\hline LVSP (mmHg) & $133 \pm 27$ & $180 \pm 27^{(*)}$ & $129 \pm 25^{(a)}$ & $152 \pm 23$ & $182 \pm 24^{(\mathfrak{b})^{(* *)}}$ \\
\hline LVEDP (mmHg) & $7 \pm 2$ & $13 \pm 2^{(*)}$ & $7 \pm 2^{(a)}$ & $10 \pm 2$ & $14 \pm 3^{\left.(\mathfrak{b})^{* * *}\right)}$ \\
\hline $\mathrm{SD}(\mathrm{mm})$ & $23.2 \pm 4.5$ & $28.1 \pm 4.4^{(*)}$ & $21.8 \pm 4.9^{(\mathrm{a})}$ & $23.1 \pm 4.3$ & $24.8 \pm 4.5^{\left.(\text {b) })^{* * 4}\right)}$ \\
\hline $\mathrm{DD}(\mathrm{mm})$ & $32.2 \pm 4.3$ & $35.1 \pm 5.0^{(*)}$ & $30.9 \pm 4.4^{(\mathrm{a})}$ & $32.4 \pm 4.4$ & $34.8 \pm 4.2^{\left.(\mathrm{b})^{(* *)}\right)}$ \\
\hline $\mathrm{Sh}(\mathrm{mm})$ & $10.6 \pm 1.4$ & $8.4 \pm 1.4^{(*)}$ & $10.8 \pm 1.0^{(\mathrm{a})}$ & $10.3 \pm 1.4$ & $9.6 \pm 1.2^{(\mathrm{b})^{(* *)}}$ \\
\hline $\mathrm{Dh}(\mathrm{mm})$ & $8.3 \pm 1.2$ & $7.0 \pm 1.2^{(*)}$ & $8.7 \pm 0.9^{(a)}$ & $7.7 \pm 0.8$ & $7.0 \pm 0.7^{(b)(* *)}$ \\
\hline \multicolumn{6}{|c|}{$\begin{array}{l}\text { To- basal level of the TAH protocol; Tm- time of maximum systolic pressure in the TAH protocol; Ho- basal level of the SAH protocol; H1- hypertensive condition } \\
1 \text { of the SAH protocol; H2- hypertensive condition } 2 \text { of the SAH protocol; a- with no statistical significance ( }>0.05) \text { in regard to the To condition of the TAH } \\
\text { protocol; b- with no statistical significance ( }>>0.05 \text { ) in regard to the Tm condition of the TAH protocol; * significantly different }(\mathrm{p}<0.05) \text { from To; **: significantly } \\
\text { different from Ho. } \mathrm{N}=13 \text { dogs. }\end{array}$} \\
\hline
\end{tabular}

termines the straight line whose angular coefficient defines the end-systolic pressure-diameter relation value.

Table II shows the individual values and the medians of ESPDR and the Pearson correlation coefficients (r) between the end-systolic points of the cardiac cycles that defined the straight lines determining ESPDR in all dogs studied. Obtaining the ESPDR index assumes the existence of linearity between the end-systolic points used for determining each of these straight lines. In our study, r values ranged from 0.913 to 0.999 . Our results are similar to those reported by other authors ${ }^{34-36}$. Therefore, we consider that the linearity condition between the end-systolic points used to obtain the straight lines determining the ESPDR values was met. Statistical analyses regarding ESPDR showed that the ESPDR-SE values are significantly higher than those of the ESPDR-TE $(p<0.05)$. Given that the variation in cardiac load was similar for both protocols, it is evident that transient and sustained elevations in blood pressure result in different ESPDR values.

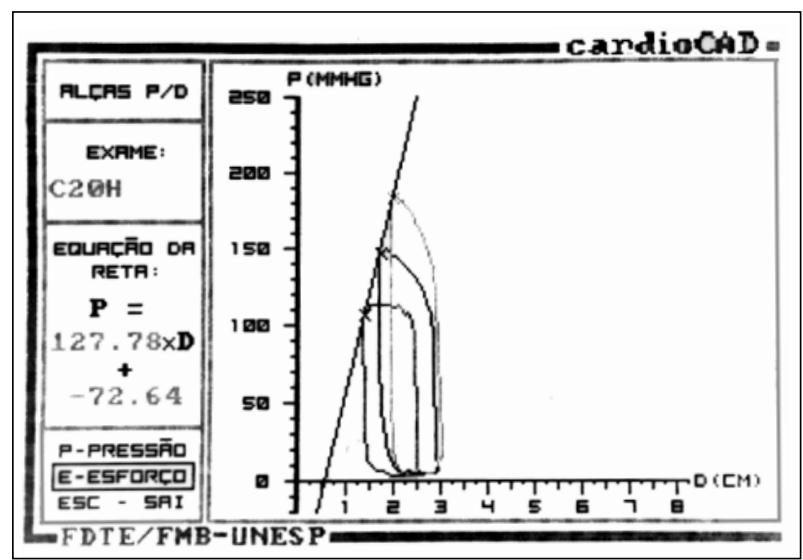

Fig. 4-Graphic representation of the pressure/diameter loops obtained during the experiments. The end-systolic points are marked with an X. The linear relation between these points determines the straight line whose angular coefficient defines the ESPDR value. P-pressure in millimeters of mercury; D- diameter in centimeters.

\begin{tabular}{|c|c|c|c|c|}
\hline \multicolumn{5}{|c|}{$\begin{array}{l}\text { Table II - Values of left ventricular end-systolic pressure-diameter } \\
\text { relation (ESPDR) obtained in the transient arterial hypertension } \\
\text { protocol (ESPDR-TE) and in the sustained arterial hypertension } \\
\text { protocol (ESPDR-SE). The Pearson correlation coefficients (r) of } \\
\text { the straight lines determining the ESPDR values are also shown. }\end{array}$} \\
\hline $\operatorname{Dog} \mathrm{N}^{\circ}$ & $\begin{array}{l}\text { ESPDR-TE } \\
(\mathrm{mmHg} / \mathrm{mm})\end{array}$ & $\mathrm{r}$ & $\begin{array}{l}\text { ESPDR-SE } \\
(\mathrm{mmHg} / \mathrm{mm})\end{array}$ & $\mathrm{r}$ \\
\hline 1 & 8.7 & 0.997 & 30.5 & 0.927 \\
\hline 2 & 9.2 & 0.993 & 16.7 & 0.983 \\
\hline 3 & 8.9 & 0.913 & 11.8 & 0.998 \\
\hline 4 & 7.6 & 0.997 & 17.9 & 0.999 \\
\hline 5 & 9.8 & 0.948 & 38.0 & 0.974 \\
\hline 6 & 11.1 & 0.979 & 15.6 & 0.932 \\
\hline 7 & 7.3 & 0.978 & 18.8 & 0.936 \\
\hline 8 & 7.4 & 0.983 & 16.1 & 0.946 \\
\hline 9 & 7.8 & 0.993 & 23.0 & 0.998 \\
\hline 10 & 9.6 & 0.959 & 24.0 & 0.917 \\
\hline 11 & 6.3 & 0.952 & 13.6 & 0.998 \\
\hline 12 & 10.9 & 0.949 & 19.8 & 0.996 \\
\hline 13 & 11.1 & 0.989 & 27.1 & 0.971 \\
\hline Median & 8.9 & - & $18.8^{*}$ & - \\
\hline $\bar{x}$ & 8.9 & 0.959 & $21.0^{*}$ & 0.967 \\
\hline sd & 1.5 & 0.031 & 7.3 & 0.031 \\
\hline \multicolumn{5}{|c|}{$\begin{array}{l}\mathrm{MmHg} / \mathrm{mm} \text { - millimeters of mercury/millimeter; }{ }^{*} \text { : significantly different } \\
(\mathrm{p}<0.05) \text { from ESPDR-TE. Values of ESPDR-TE and ESPDR-SE obtained } \\
\text { with an increase of } 50 \mathrm{mmHg} \text { in blood pressure. }\end{array}$} \\
\hline
\end{tabular}

\section{Discussion}

In this study, the values of the variables indicating the preload (DD; LVEDP; Dh) and afterload (SD; LVSP; Sh) conditions significantly increased, and they were equivalent when blood pressure reached its maximum value (Tm and $\mathrm{H} 2$ ). Therefore, the fundamental characteristic differentiating transient and sustained arterial hypertension protocols was the duration of pressure elevation. Therefore, the ESPDR results allow us to consider that the most significant finding of this study was that transient and sustained blood pressure elevations are followed by time-dependent cardiac adjustments capable of increasing the ESPDR values.

In experimental conditions, the viscoelastic myocardial 
accommodations, the alterations in afterload, and the accentuation in cardiac inotropism should be considered as factors potentially capable of interfering with and modifying the ESPDR values during pressure elevations.

In regard to viscoelastic accommodations, some studies ${ }^{37-39}$ that assessed these properties in in situ hearts applying pressure or volume overloads within physiological limits did not identify alterations in left ventricular P/V relations compatible with viscoelastic accommodations. Time-dependent deviations of left ventricular P/V relations in dogs were shown only when marked pressure (LVSP $>200 \mathrm{mmHg}$ ) or volume $\left(\right.$ LVEDP $>25 \mathrm{mmHg}$ ) overloads were induced ${ }^{40}$. Way et al $^{41}$ assessed the influence of viscoelastic myocardial accommodations upon $\mathrm{P} / \mathrm{V}$ relations in in situ hearts of dogs undergoing beta-adrenergic blockade and thoracotomy. In that study, the authors did not find alterations in the ESPDR values when elevation or reduction in the blood pressure occurred within 4 to 6 heartbeats and LVEDP was normal or increased. In our study, variations in cardiac load did not reach the values reported ${ }^{40}$ as capable of triggering significant changes in the $\mathrm{P} / \mathrm{V}$ relations depending on viscoelastic accommodations. According to the literature ${ }^{40,41}$, the deviations resulting from viscoelastic accommodations tend to reduce ESPDR values, which opposes the variations observed in our study. Therefore, if elevations in blood pressure were accompanied by viscoelastic myocardial accommodations, they worked reducing the increases in the ESPDR promoted by other dominating factors.

Given that in this study the ESPDR was determined during primary changes in afterload, we should consider that the differences in the ejecting ventricular pattern during blood pressure elevation might have interfered with the results obtained. Several studies assessing the behavior of ESPDR during afterload elevations in preparations of isolated ${ }^{42,43}$ and in situ ${ }^{44,45}$ hearts concluded that this contractility index does not significantly change during pressure elevations. Freeman et al $^{45}$ assessed the ESPDR values in preparations of in situ heart, where the heart ejected blood into the native aorta or into a rigid arterial system. In this last study, the authors showed that acute changes in vascular compliance do not significantly change the ESPDR values. Taking all this evidence in the literature into consideration, it seems reasonable to conclude that the afterload level and characteristics do not directly interfere with the assessments of ESPDR and, therefore, in our study, changes in the ESPDR values did not depend on changes in pressure levels. Given that in the in situ heart a constant interaction between preload and afterload ${ }^{24}$ exists and that the ESPDR-SE values were significantly more elevated than those of ESPDR-TE (table II), it is clear that sustained blood pressure elevations triggered time-dependent cardiac adjustments that stressed cardiac inotropism. Analyzing cardiac adaptations during pressure elevations in such conditions, the variations in heart rate, the changes in the coronary perfusion pressure (CPP), and the changes in the ventricular volume depending on the interaction between preand afterload deserve to be highlighted.

In regard to heart rate, several studies ${ }^{3,36,46,47}$ report an increase in ESPDR values associated with an elevation in the heart rate. Data analysis related to heart rate (table I) shows stability of the cardiac automatism during the experiments. These results assure that heart rate did not play a role in the oscillations of inotropism and, therefore, the ESPDR values did not result from fluctuations in heart rate.

Coronary artery perfusion may affect the heart contractile capacity due to biochemical or physical reasons. Biochemical influences that may result from CPP are those traditionally linked to ischemia. In experimental conditions, a specific pattern of myocardial ischemia has to be considered: the one related to the Anrep effect. This effect consists of the fluctuation of inotropism following sudden elevations in ventricular systolic pressure ${ }^{48-50}$. This sudden elevation in ventricular pressure causes the subendocardial vessels to collapse with ischemia and depression of the local muscular contraction. Then, a quick normalization of blood flow and recovery of the inotropic state occur due to the feedback coronary mechanism. These inotropic oscillations linked to the Anrep effect are established in a few seconds. On the other hand, it was recently proposed that elevations in CPP might intensify the influx of calcium for the myocytes, constituting another interfering factor with myocardial inotropism ${ }^{51}$. It is worth adding that the relations between perfusion pressure and ventricular performance are simultaneously established with the variation in pressure, being complete after a few heartbeats.

Physical influences of CPP on left ventricular performance were shown in situations where metabolic factors could be considered as nonparticipating ${ }^{52-55}$. CPP is admitted to influence ventricular mechanical action modulating the length of fibers overlapping the coronary arteries ${ }^{52-55}$ and interfering with the dimensions of the ventricular wall ${ }^{54}$. Either by one reason or another, coronary perfusion may regulate fiber length independently from the cavity volume.

The effects of CPP on ESPDR values have already be-

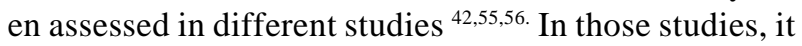
was shown that ESPDR is not sensitive to variations in CPP when the perfusion pressure is $>60 \mathrm{mmHg}$. In our study, LVSP (left ventricular systolic pressure) was always $>100 \mathrm{mmHg}$ and, therefore, no reason exists for supposing that CPP had effects on the results obtained.

In the in situ heart, variations in the ventricular volume occurring during hypertension are established due to forces of interaction between the pre- and afterloads ${ }^{24,57,58}$. The increases in afterload are followed by a reduction in the ejected volume in the subsequent systoles, and the amount of residual blood remaining in the ventricular cavity causes dilation in the chamber. This functional sequence is one of the classic vias for activating the Frank-Starling mechanism ${ }^{59}$. The research ${ }^{18-21}$ that analyzed the physiological bases of this mechanism confirmed that the accentuation of the contractile capacity caused by myocardial stretching includes changes in the cellular kinetics of the calcium ion as well as in the affinity of its ligation with contractile proteins. Therefore, among the subcellular adjustments caused by myocardial 
stretching, adaptations of the biochemical process of contraction exist, characterizing an inotropic intervention. The subcellular rearrangements linked to myocardial stretching begin simultaneously with variations in the cellular length, being completed after a few minutes ${ }^{20,21,55}$. In our study, it was evident that the elevations in blood pressure were accompanied by significant increases in left ventricular diastolic dimensions, characterizing, therefore, a maneuver of increase in the ventricular volume with consequent myocardial stretching. Currently, it is understood that during a sustained elevation of pressure levels for 10 minutes, as occurred in the sustained arterial hypertension protocol, cardiac accommodations associated with hypertension have already been completed making, in this way, the occurrence of inotropism activation possible. The existence of a linear relation between the values of left ventricular diastolic dimensions and the first temporal derivative $(\mathrm{dP} / \mathrm{dT})$ with a strong relation to the contractile state ${ }^{60}$ has already been reported.

Considering data available the results observed in our study, it may be concluded that the ESPDR values determined in the sustained arterial hypertension protocol are higher than those verified in the transient arterial hypertension protocol. The major mechanism most probably involved in inotropic activation is the time-dependent activation caused by adaptations of the biochemical process of contraction, characterizing an inotropic intervention. The subcellular rearrangements related to myocardial stretching begin simultaneously with variations in length and are completed in a few minutes ${ }^{19-23}$. This interpretation in accordance with data available in the literature about cardiac accommodations during pressure elevations justifies the use of ESPDR as an index of myocardial contractility in stable physiological conditions. It is worth clarifying that data from this study do not allow the assessment of the relative contribution of coronary perfusion pressure and ventricular volume for the increases in the ESPDR values when this inotropic index is determined during sustained arterial hypertensions.

\section{Acknowledgments}

We thank Dr. Marina Politi Okoshi for her support.

\section{References}

1. Suga, H. Left ventricular pressure volume ratio in systole as an index of myocardial inotropism. Jpm Heart J 1971; 12:153-160.

2. Suga H, Sagawa K, Shoukas AA. Load-independence of instantaneouspressure-volume ratio of the canine ventricle and effects of epinephrine and heart rate on the ratio. Circ Res 1973; 32: 314-22.

3. Suga $\mathrm{H}$, Sagawa K. Instantaneous pressure-volume relationships and their ratio in the excised, supported canine left ventricle. Circ Res 1974; 35: 117-26.

4. Suga H, Yamakoshi KI. Effects of stroke volume and velocity of ejetion on endsystolic pressure of canine left ventricle: Endy-systolic volume clamping. Circ Res 1977; 40: 445-50.

5. Sagawa K, Shoukas AA, Bakalar KM. End-systolic pressure-volume ratio. A new index of contratility. Am J Cardiol 1977; 40: 748-53.

6. Mahler F, Covell JW, Ross J Jr. Systolic pressure-diameter relations in the normal conscious dogs. Cardiovasc Res 1975; 35: 447-55.

7. Maughan WH, Sunagawa K. Factors affecting the end-systolic pressure-volume relationship. Fed Proc 1984; 43: 2408-10.

8. Maughan, WH, Sunagawa K, Burkhoff D, Sagawa K. Effect of arterial impedance changes on the end-systolic pressure-volume relation. Circ Res 1984; 54: 595-602.

9. Nishioka O, Maruyama Y, Ashikawa K, et al. Load dependency of end-systolic pressure-volume relations in isolated ejecting canine hearts. Jpm Heart J 1988; 29: 709-22.

10. Hunter WC. End-systolic pressure as balance between opposing effects of ejetion. Circ Res 1989; 64: 265-75.

11. Su JB, Crozatier B. Preload-induce curvilinearity of left ventricular end-systolic pressure-volume relations. Effects on derived indexes in closed chest dogs. Circulation 1998; 79: 431-40.

12. Sugiura S, Hunter WC, Sagawa K. Long-term versus intrabeat history of ejection as determinants of canine ventricular end-systolic pressure. Circ Res 1989; 64: 255-64

13. Van der VeldET, BurkhoffD, Steendij KP, Kardson J, Sagawa K, Baan J. Non linearity and load sensitivity of end-systolic pressure-volume relation on canine left ventricle in vivo. Circulation 1991; 83: 315-27.

14. Crottogini AJ, Willshaw P, Barra JA, Pichel RH. Left ventricular end-systolic elastance is incorrectly estimated by the use of variations in conscious, unsedated, autonomically intact dogs. Circulation 1994; 90: 1431-40.

15. Burkhoff D, Sugiura S, Yue D, Sagawa K. Contratility-dependent curvilinearity of end-systolic pressure-volume relations. Am J Physiol 1987; 252: H218-H26.

16. Parmley WW, Chuck L. Length-dependence changes in myocardial contractile state. Am J Physiol 1973; 224: 1195-9.
17. Lakata, EG, Jeweel. Length-dependence activation. Its effect on the lenghttension relation in cat ventricular muscle. Circ Res 1977; 40: 251-7.

18. Allen DG, Kentish JC. The cellular basis on the lenght-tension relation in cardiac muscle. J Moll Cell Cardiol 1985; 17: 821-40.

19. Lakata EG. Starling's law of the heart is explained by an intimate interaction of muscle lenght and myofilament calcium activation. J Am Coll Cardiol 1987; 10 1157-64.

20. Kentish JC. The lenght tension relation in the myocardial and its cellular basis Heart Failure 1988; 4: 125-36.

21. Tucci PJF, BregagnolloEA, Spadaro J, Cicogna AC, Ribeiro MCL. Lenght dependence of activation studied in the isovolumic blood-perfused dog heart. Circ Res 1984; 55: 59-66.

22. Stefanon I, Vassalo DV, Mill JC. Left ventricular length denpendent activation in the isovolumetric rat heart. Cardiovasc Res 1990; 24: 254-6.

23. Lew WYW. Time-dependence increase in left ventricular contractility following acute volume loading in dog. Circ Res 1988; 63: 635-47.

24. Matsubara BB, Tucci PJF. A interação entre a pré e a pós-carga. Os desajustes da pós-carga: implicações clínicas. Arq Bras Cardiol 1987; 48: 135-6.

25. Matsubara BB, BregagnolloEA, Tucci PJF, et al. Left ventricular maximal systolic elastance calculated by a combination of M-mode echocardiography and standard manometry. Braz J Med Biol Res 1991; 24: 383-94.

26. O'Rourke RA, Hanrath P, Henry WN, et al. Report of the joint International Society and Federation of Cardiology/World Health Organization Task Force on recommendations for standardization of measurements from M-Mode echocardiograms. Circulation 1984; 69: 854-7.

27. BregagnolloEA. A elastância sistólica final do ventrículo esquerdo determinada durante elevações transitórias e elevações sustentadas da pressão arterial. Tese de doutorado. Faculdade de Medicina de Botucatu - UNESP, 1991.

28. Shroff SG, Weber KT, Janicki JS. End-systolic relations: their usefulness and limitations in assessing left ventricular contractile state. Int J Cardiol 1984; 5: 253-9.

29. Ostle B. Estatística Aplicada. México: Limusa-Willy, 1973: 629.

30. Siegen S. Nonparametric Statistic for Behavioral Sciences. $1^{\text {a }}$ ed. New York: McGraw-Hill Book Co., 1956: 350p.

31. Mahler F, Ross J Jr, O'Rourke RA, et al. Effects of changes in preload, afterload and inotropic state of ejection and isovolumic phase measures of contratility in the conscious dogs. Am J Cardiol 1975; 35: 626-38.

32. Crozatier B, Caillet D, Thuillez C, et al. Preload changes modify systolic pressure diameter relations in the conscious dogs. Am J Physiol 1981; 240: 354-60. 
33. Sasayma S, Franklin D, Ross J Jr. Hiperfunction with normal inotropic state of the hypertrophied left ventricle. Am J Physiol 1977; 1: 418-25.

34. Igarashi Y, Suga H. Assessment of the end-systolic pressure-volume line of in situ dog heart. Am J Physiol 1986; 25: H685-H92.

35. Little WC, Freedman GL. Description of LV pressure-volume relations by time-varying elastance and source resistance. Am J Physiol 1987; 253: H83H8.

36. Sprat JA, Tyson GS, Glower DD, et al. The end-systolic pressure-volume relationship in conscious dogs. Circulation 1987; 75: 1295-309.

37. Noble MIM, Milne ENC, Goerke RJ, et al. Left ventricular filling and diastolic pressure-volume in the conscious dogs. Circ Res 1969; 24: 269-83.

38. Ran King JS, Aretzen CE, McHale PE, et al. Determination of left ventricular preload and afterload by quantitative echocardiography in man. Calibration of the method. Circ Res 1974; 34: 711-18.

39. Glantz AS, Parmley WW. Factors wich affect the diastolic pressure-volume curve. Circ Res 1980; 42: 171-80.

40. Le-WinterMM,EnglerR, Pavalec RS. Time-dependente shifts on the left ventricular diastolic filling relationship in conscious dogs. Circ Res 1979; 45: 641-53.

41. Way B, Victory J, Le-Winter MM, et al. Hysteresis of left ventricular and ejection pressure-dimension relations after acute pressure loading in the intact canine heart. Cardiovasc Res 1986; 20: 490-7.

42. Maughan WH, Sunagawa K. Factor affecting the end-systolic pressure-volume relationship. Fed Proc 1984; 43: 2408-10.

43. Maughan WH, Sunagawa K, Burkoff D, et al. Effect of arterial evidence changes on the end-systolic pressure-volume relation. Circ Res 1984; 54: 595-602.

44. Spratt JA, Tyson GS, Glower DD, et al. The end-systolic pressure-volume relationship in the concious dogs. Circulation 1987; 75: 1295-309.

45. Freeman GL, Little WC, Rourke RA. The effect of vasoative agents on the left ventricular end-systolic pressure-volume relation in closed chest dogs. Circulation 1986; 74: 1107-13.

46. Aliono D, Larson EV, Crumbley AJ, et al. Is Emax independent of heart rate in the conscious dogs. Circulation 1982; 66: 347-8.
47. Maughan WH, Sunagawa K, BurkoffD, et al. Effect of heart rate on the canine endsystolic pressure-volume relationship. Circulation 1985; 72: 654-9.

48. Monroe RG, Ganble WJ, LaFarge GG, et al. The Anrep effect reconsiderece. J Clin Invest 1972; 51: 2573-83.

49. Vatner SF, Monroe RG, McRitchie RJ. Effects of anesthesia, tachycardia and autonomic blockade on the Anrep effect in intact dogs. Am J Physiol 1974; 226: 1450-6.

50. Walston A, Rembertj C, Fedor JM, et al. Regional myocardial blood flow after sudden aortic constriction in awake dogs. Circ Res 1978; 42: 419-25.

51. Kitakaze M, Marban E. Cellular mechanism of the modulation of contractile function by coronary perfusion pressure in ferret hearts. J Physiol 1989; 414: 455-72.

52. Opie LH. Coronary flow rate and perfusion pressure as a determinants of mechanical function and oxidative metabolism of isolated perfused rat heart. Physiol 1965; 180: 529-41.

53. Benekenj E, Guyton AC, Sagawa K. Coronary perfusion on pressure and left ventricular function. Pflügers Arch 1969; 305: 76-95.

54. Tucci, PJF, BregagnolloEA, Spadaro J, Cicogna AC. Coronary perfusion pressure as a determinant of ventricular performance. Experientia 1980; 36:974-5.

55. AbelRM, Reis RL. Effects of coronary blood flow and perfusion pressure on left ventricular contractility in dogs. Circ Res 1970; 27: 961-71.

56. Sunagawa K, Maughan KW, Friesinger G, et al. The effects of coronary arterial pressure on ventricular end-systolic pressure-volume relation of isolated canine heart. Circ Res 1982; 50: 727-34.

57. Ross J Jr, Covel JW, Sonnenblick EH. Contractile state of heart caracterized by force/velocity relations in variably afterload and isovolumic beats. Circ Res 1966; 18: 149-63.

58. Lee JD, Tajimi T, Patritti J, et al. Preload reserve and mechanism of afterload nismatch in normal conscious dogs. Am J Physiol 1986; 250: H464-H8.

59. Petterson SW, Pipper H, Starling EA. The regulation of the heart beat. J Physiol (Lond) 1914; 48: 463-513.

60. Little WC. The left ventricular dp/dt max - end-diastolic-volume relation in closed-chest dogs. Circ Res 1985; 56: 808-15. 